\title{
THE KINETIC ENERGY FORMULA FOR THE CLOSED PLANAR HOMOTHETIC MOTIONS IN COMPLEX PLANE
}

\author{
A. $\operatorname{Tutar}^{1 \S}$, O. Sener ${ }^{2}$ \\ ${ }^{1,2}$ Department of Mathematics \\ Ondokuz Mayis University \\ 55139 Kurupelit, Samsun, TURKEY
}

\begin{abstract}
In this paper, the kinetic energy formula was expressed during one-parameter closed planar homothetic motions in complex plane. Then the relation between the kinetic energy formula and the Steiner formula was given. As an example the sagittal motion of a winch was considered. This motion was described by a double hinge consisting of the fixed control panel of winch and the moving arm of winch. The results were applied to experimentally measured motion.
\end{abstract}

AMS Subject Classification: 53A17, 70B10

Key Words: kinetic energy, Steiner formula, complex plane, homothetic motions

\section{Introduction}

For a geometrical object rolling on a line and making a complete turn, some properties of the area of a path of a point were given by [6]. The Steiner area formula and the Holditch theorem during one parameter closed planar homothetic motions were expressed by [7]. If the points of the moving plane which enclose the same area lie on a circle, then the centre of this circle is called the Steiner point $(h \equiv 1)[4,5]$. Dathe and Gezzi expressed the Steiner area formula and the polar moment of inertia for the closed planar motions [1]. The

Received: March 30, 2015 (C) 2015 Academic Publications

$\S$ Correspondence author 
study reported by Dathe and Gezzi [1] was investigated during one- parameter closed planar homothetic motions in complex plane by Tutar and Sener [8]. In the case of the homothetic scale $h \equiv 1$ the results given by [1] were obtained as a special case. Dathe and Gezzi expressed the formula of kinetic energy for the closed planar kinematics [2]. Furthermore, the expressions reported by [2] were examined under one-parameter closed planar homothetic motions by [3]. In this paper, the expressions given by [3] were researched in complex plane. Furthermore, we expressed the relation between the area enclosed by a path and the kinetic energy. In the case of the homothetic scale $h \equiv 1$ the results given by [2] were obtained as a special case. As an example, Dathe and Gezzi have chosen the sagittal part of the movement of the human leg during walking for planar kinematics [1]. We considered the sagittal motion of a telescopic crane which is described by a double hinge being fixed and moving as an example. The kinetic energy formula was calculated for this motion. Moreover, the relation between the kinetic energy and the Steiner formula was expressed.

\section{The Kinetic Energy for the Closed Planar Homothetic Motion}

We consider one parameter closed planar homothetic motion between two reference systems: the fixed $E^{\prime}$ and the moving $E$, with their origins $\left(O, O^{\prime}\right)$ and orientations in complex plane. Then, we take into account motion relative to the fixed coordinate system (direct motion).

By taking displacement vectors $O O^{\prime}=U^{\prime}$ and $O^{\prime} O=U$ the total angle of rotation $a(t)$, the motion defined by the transformation

$$
X^{\prime}(t)=h(t) X e^{i a(t)}+U^{\prime}(t)
$$

is called one-parameter closed planar homothetic motion and denoted by $E / E^{\prime}$, where $h$ is a homothetic scale of the motion $E / E^{\prime}, X$ and $X^{\prime}$ are the position vectors with respect to the moving and fixed rectangular coordinate systems of a point $X \in E$, respectively. The homothetic scale $h$ and the vectors $X^{\prime}$ and $U, U^{\prime}$ are continuously differentiable functions of a real parameter $t$.

In Eq. (1), $X^{\prime}(t)$ is the trajectory with the respect to the fixed system of a point $X$ belonging to the moving system. If we replace $U^{\prime}=-U e^{i a(t)}$ in Eq. (1), the motion can be written as

$$
X^{\prime}(t)=(h(t) X-U(t)) e^{i a(t)}
$$


The coordinates of the above equation are

$$
\begin{aligned}
X^{\prime}(t) & =x_{1}^{\prime}(t)+i x_{2}^{\prime}(t), \quad U^{\prime}(t)=u_{1}^{\prime}(t)+i u_{2}^{\prime}(t) \\
X & =x_{1}+i x_{2}, \quad U(t)=u_{1}(t)+i u_{2}(t) .
\end{aligned}
$$

Using these coordinates, we can write

$$
\begin{aligned}
x_{1}^{\prime}(t)+i x_{2}^{\prime}(t)= & {\left[\left(h(t) x_{1}-u_{1}(t)\right)+i\left(h(t) x_{2}-u_{2}(t)\right)\right] } \\
& \times(\cos (a(t))+i \sin (a(t))) .
\end{aligned}
$$

From Eq. (3), the components of $X^{\prime}(t)$ may be given as

$$
\begin{aligned}
& x_{1}^{\prime}(t)=\cos a(t)\left(h(t) x_{1}-u_{1}(t)\right)-\sin a(t)\left(h(t) x_{2}-u_{2}(t)\right), \\
& x_{2}^{\prime}(t)=\sin a(t)\left(h(t) x_{1}-u_{1}(t)\right)+\cos a(t)\left(h(t) x_{2}-u_{2}(t)\right) .
\end{aligned}
$$

Using the coordinates of Eq. (2) as

$$
X^{\prime}(t)=\left(\begin{array}{l}
x_{1}^{\prime}(t) \\
x_{2}^{\prime}(t)
\end{array}\right), U^{\prime}(t)=\left(\begin{array}{l}
u_{1}^{\prime}(t) \\
u_{2}^{\prime}(t)
\end{array}\right), X=\left(\begin{array}{l}
x_{1} \\
x_{2}
\end{array}\right), U(t)=\left(\begin{array}{l}
u_{1}(t) \\
u_{2}(t)
\end{array}\right)
$$

and rotation matrix

$$
R(t)=\left(\begin{array}{cc}
\cos (a(t)) & -\sin (a(t)) \\
\sin (a(t)) & \cos (a(t))
\end{array}\right)
$$

we can obtain

$$
X^{\prime}(t)=R(t)(h(t) X-U(t))
$$

If we differentiate Eq. (4), we have

$$
\begin{aligned}
\dot{x}_{1}^{\prime}= & -\left(h x_{1}-u_{1}\right) \sin a \cdot \dot{a}+\left(\dot{h} x_{1}-\dot{u_{1}}\right) \cos a \\
& -\left(h x_{2}-u_{2}\right) \cos a \cdot \dot{a}-\left(\dot{h} x_{2}-\dot{u_{2}}\right) \sin a \\
\dot{x}_{2}^{\prime}= & \left(h x_{1}-u_{1}\right) \cos a \cdot \dot{a}+\left(\dot{h} x_{1}-\dot{u_{1}}\right) \sin a \\
& -\left(h x_{2}-u_{2}\right) \sin a \cdot \dot{a}+\left(\dot{h} x_{2}-\dot{u_{2}}\right) \cos a .
\end{aligned}
$$

A moment with a first order in the time derivatives can be introduced by

$$
S=\frac{1}{2} \int\left[\left(\dot{x}_{1}^{\prime}\right)^{2}+\left(\dot{x}_{2}^{\prime}\right)^{2}\right] d t
$$


which is the integral over the kinetic energy of a point with mass $M=1$.

Using Eq. (9)

$$
\begin{aligned}
\left(\dot{x}_{1}^{\prime}\right)^{2}+\left(\dot{x}_{2}^{\prime}\right)^{2}= & h^{2}\left(x_{1}^{2}+x_{2}^{2}\right) \dot{a}^{2}+\left(x_{1}^{2}+x_{2}^{2}\right) \dot{h}^{2} \\
& +\left(u_{1}^{2}+u_{2}^{2}\right) \dot{a}^{2}+\dot{u}_{1}^{2}+\dot{u}_{2}^{2}+2 u_{1} \dot{u}_{2} \dot{a}-2 u_{2} \dot{u}_{1} \dot{a} \\
& +x_{1}\left(-2 h u_{1} \dot{a}^{2}-2 \dot{h} \dot{u}_{1}-2 h \dot{u}_{2} \dot{a}+2 \dot{h} u_{2} \dot{a}\right) \\
& +x_{2}\left(-2 h u_{2} \dot{a}^{2}-2 \dot{h} \dot{u}_{2}+2 h \dot{u}_{1} \dot{a}-2 \dot{h} u_{1} \dot{a}\right)
\end{aligned}
$$

is obtained. If Eq. (11) is replaced in Eq. (10), we have

$$
\begin{aligned}
2 S= & \left(x_{1}^{2}+x_{2}^{2}\right) \int\left(h^{2} \dot{a}^{2}+\dot{h}^{2}\right) d t \\
& +\int\left[\left(u_{1}^{2}+u_{2}^{2}\right) \dot{a}^{2}+\dot{u}_{1}^{2}+\dot{u}_{2}^{2}+2 u_{1} \dot{u}_{2} \dot{a}-2 u_{2} \dot{u}_{1} \dot{a}\right] d t \\
& +x_{1} \int\left[-2 h u_{1} \dot{a}^{2}-2 \dot{h}_{\dot{u}_{1}}-2 h \dot{u}_{2} \dot{a}+2 \dot{h} u_{2} \dot{a}\right] d t \\
& +x_{2} \int\left[-2 h u_{2} \dot{a}^{2}-2 \dot{h} \dot{u}_{2}+2 h \dot{u}_{1} \dot{a}-2 \dot{h} u_{1} \dot{a}\right] d t .
\end{aligned}
$$

If $X=O \quad\left(x_{1}=x_{2}=0\right)$ is taken, then, for the formula of kinetic energy of the origin point

$$
2 S_{O}=\int\left[\left(u_{1}^{2}+u_{2}^{2}\right) \dot{a}^{2}+\dot{u}_{1}^{2}+\dot{u}_{2}^{2}+2 u_{1} \dot{u}_{2} \dot{a}-2 u_{2} \dot{u}_{1} \dot{a}\right] d t
$$

is found. If Eq. (13) is replaced in Eq. (12),

$$
\begin{aligned}
2\left(S-S_{O}\right)= & \left(x_{1}^{2}+x_{2}^{2}\right) \int\left(h^{2} \dot{a}^{2}+\dot{h}^{2}\right) d t \\
& +x_{1} \int\left[-2 h u_{1} \dot{a}^{2}-2 \dot{h} \dot{u}_{1}-2 h \dot{u}_{2} \dot{a}+2 \dot{h} u_{2} \dot{a}\right] d t \\
& +x_{2} \int\left[-2 h u_{2} \dot{a}^{2}-2 \dot{h} \dot{u}_{2}+2 h \dot{u}_{1} \dot{a}-2 \dot{h} u_{1} \dot{a}\right] d t
\end{aligned}
$$

is obtained.

Now we consider the case in which the motion is closed and naturally parametrized. Then, it follows 


$$
\begin{aligned}
d t \rightarrow d a, \dot{a} \rightarrow a^{\prime}= & \frac{d a}{d a}=1 \text { and } \int \rightarrow \oint \text {. With those assumptions, we obtain } \\
2\left(S-S_{O}\right)= & x_{1} \oint\left[-2 h u_{1}-2 d h d u_{1}-2 h d u_{2}+2 d h u_{2}\right] d a \\
& +x_{2} \oint\left[-2 h u_{2}-2 d h d u_{2}+2 h d u_{1}-2 d h u_{1}\right] d a \\
& +\left(x_{1}^{2}+x_{2}^{2}\right) \oint\left(h^{2}+(d h)^{2}\right) d a
\end{aligned}
$$

Eq. (18) of [8] is as below: $U=\left(\begin{array}{l}u_{1} \\ u_{2}\end{array}\right)$,

$$
\begin{aligned}
& u_{1}=h p_{1}+p_{2} \frac{d h}{d a}-\frac{d u_{2}}{d a}, \\
& u_{2}=h p_{2}-p_{1} \frac{d h}{d a}+\frac{d u_{1}}{d a} .
\end{aligned}
$$

If Eq. (18) of [8] is replaced at coefficients of $x_{1}$ and $x_{2}$ in Eq. (15), respectively and by calculating necessary operations,

$$
\begin{aligned}
2\left(S-S_{O}\right)= & x_{1} \oint\left[-2 h^{2} p_{1} d a-2 h p_{2} d h-2 \frac{d h}{d a} d u_{1}+2 u_{2} d h\right] \\
& +x_{2} \oint\left[-2 h^{2} p_{2} d a+2 h p_{1} d h-2 \frac{d h}{d a} d u_{2}-2 u_{1} d h\right] \\
& +\left(x_{1}^{2}+x_{2}^{2}\right) \oint h^{2} d a+\left(x_{1}^{2}+x_{2}^{2}\right) \oint \frac{(d h)^{2}}{d a}
\end{aligned}
$$

is obtained.

If we consider Eqs. (14), (19), (20), (21) and (22) of [8]

$$
\begin{gathered}
m=\oint h^{2} d a=h^{2}\left(t_{0}\right) \oint d a=h^{2}\left(t_{0}\right) 2 \pi \nu \\
a^{*}=\oint\left(-2 h^{2} p_{1} d a\right)+\oint\left(-2 h p_{2} d h+h d u_{2}+u_{2} d h\right), \\
b^{*}=\oint\left(-2 h^{2} p_{2} d a\right)+\oint\left(2 h p_{1} d h-h d u_{1}-u_{1} d h\right), \\
a:=\oint-2 h^{2} p_{1} d a, \quad b:=\oint-2 h^{2} p_{2} d a \\
\mu_{1}:=\oint\left(-2 h p_{2} d h+h d u_{2}+u_{2} d h\right), \\
\mu_{2}:=\oint\left(2 h p_{1} d h-h d u_{1}-u_{1} d h\right)
\end{gathered}
$$


and

$$
2\left(F-F_{O}\right)=\left(x_{1}^{2}+x_{2}^{2}\right) m+a^{*} x_{1}+b^{*} x_{2}
$$

and Eqs. (14), (19), (20), (21) and (22) of [8] are replaced in Eq. (16),

$$
\begin{aligned}
2\left(S-S_{O}\right)= & 2\left(F-F_{O}\right)+\left(x_{1}^{2}+x_{2}^{2}\right) \oint \frac{(d h)^{2}}{d a} \\
& +x_{1} \oint\left(u_{2} d h-h d u_{2}-2 \frac{d h}{d a} d u_{1}\right) \\
& +x_{2} \oint\left(-u_{1} d h+h d u_{1}-2 \frac{d h}{d a} d u_{2}\right)
\end{aligned}
$$

is arrived at the relation between the formula of kinetic energy and the formula for the area.

\section{Example: The Direct Motion of Telescopic Crane}

The motion of telescopic crane has a double hinge and 'a double hinge' is mean that it has two systems, a fixed arm and a moving arm of telescopic crane (Figure 1). There is a control panel of telescopic crane at the origin of moving system. ' $\mathrm{L}$ ' arm can extend or retract by $\mathrm{h}$ parameter. Also we define using the condition $m=0$ (Eq. (15) of [8]), the Steiner line and the total angle in relation to the double hinge.

By taking displacement vectors $O O^{\prime}=U^{\prime}$ and $O^{\prime} O=U$, the total angle of rotation $l-k=a$, the motion defined by the transformation

$$
X^{\prime}(t)=h(t) X e^{i a(t)}+U^{\prime}(t)
$$

is called one-parameter closed planar homothetic motion and denoted by $E / E^{\prime}$, where $h$ is a homothetic scale of the motion $E / E^{\prime}, X$ and $X^{\prime}$ are the position vectors with respect to the moving and fixed rectangular coordinate systems of a point $X \in E$, respectively. The homothetic scale $h$ and the vectors $X^{\prime}$ and $U, U^{\prime}$ are continuously differentiable functions of a real parameter $t$.

By taking

$$
R(t)=\left(\begin{array}{cc}
\cos (l(t)-k(t)) & -\sin (l(t)-k(t)) \\
\sin (l(t)-k(t)) & \cos (l(t)-k(t))
\end{array}\right)
$$

and

$$
U(t)=\left(\begin{array}{l}
-L \cos (l(t)-k(t)) \\
-L \sin (l(t)-k(t))
\end{array}\right)
$$




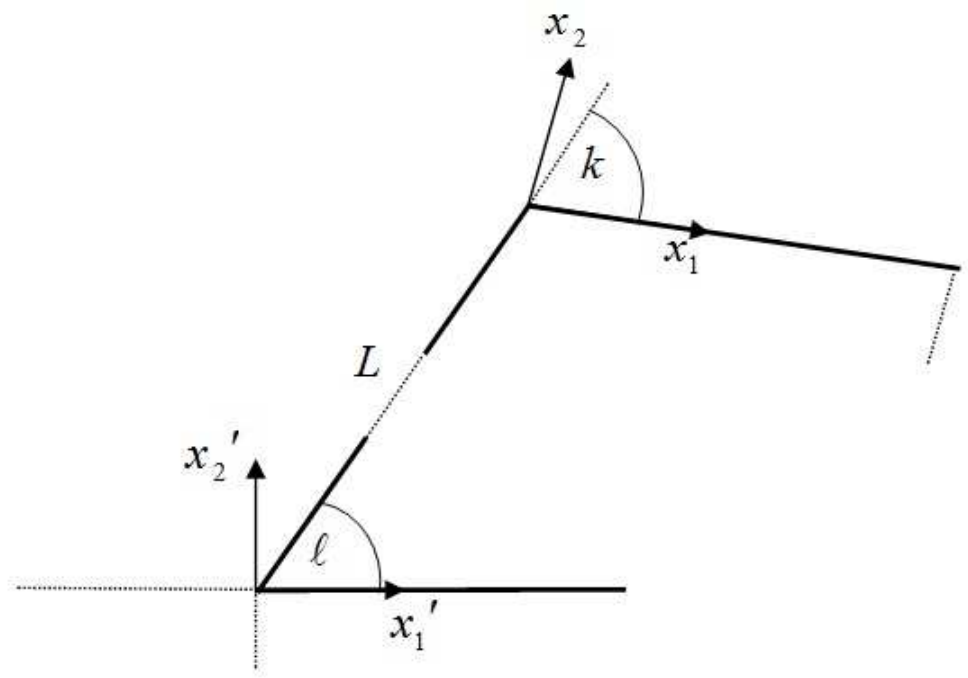

Figure 1: The arms of telescopic crane as a double hinge

we have

$$
X^{\prime}(t)=R(t)(h(t) X-U(t)) .
$$

By considering Eq. (42) of [8]

$$
\begin{aligned}
x_{1}^{\prime}(t)= & \cos (\ell(t)-k(t))\left(h(t) x_{1}+L \cos k(t)\right) \\
& -\sin (\ell(t)-k(t))\left(h(t) x_{2}+L \sin k(t)\right), \\
x_{2}^{\prime}(t)= & \sin (\ell(t)-k(t))\left(h(t) x_{1}+L \cos k(t)\right) \\
& +\cos (\ell(t)-k(t))\left(h(t) x_{2}+L \sin k(t)\right)
\end{aligned}
$$

and if we calculate the time derivative of this equation,

$$
\begin{aligned}
\dot{x}_{1}^{\prime}= & -\left(h x_{1}+L \cos k\right) \sin (\ell-k)(\dot{\ell}-\dot{k}) \\
& +\left(\dot{h} x_{1}-L \sin k \cdot \dot{k}\right) \cos (\ell-k) \\
& -\left(h x_{2}+L \sin k\right) \cos (\ell-k)(\dot{\ell}-\dot{k}) \\
& -\left(\dot{h} x_{2}+L \cos k \cdot \dot{k}\right) \sin (\ell-k),
\end{aligned}
$$




$$
\begin{aligned}
\dot{x}_{2}^{\prime}= & \left(h x_{1}+L \cos k\right) \cos (\ell-k)(\dot{\ell}-\dot{k}) \\
& +\left(\dot{h} x_{1}-L \sin k \cdot \dot{k}\right) \sin (\ell-k) \\
& -\left(h x_{2}+L \sin k\right) \sin (\ell-k)(\dot{\ell}-\dot{k}) \\
& +\left(\dot{h} x_{2}+L \cos k \cdot \dot{k}\right) \cos (\ell-k)
\end{aligned}
$$

is obtained.

If we calculate $\dot{x}_{1}^{2}+\dot{x}_{2}^{2}$ for the formula of kinetic energy and use in Eq. (10), we have

$$
\begin{aligned}
2 S= & x_{1} \int\left[2 h L \cos k(\dot{\ell}-\dot{k})^{2}-2 \dot{h} L \sin k \cdot \dot{k}\right. \\
& +2 h L \cos k \cdot \dot{k}(\dot{\ell}-\dot{k})-2 \dot{h} L \sin k(\dot{\ell}-\dot{k})] d t \\
& +x_{2} \int\left[2 h L \sin k(\dot{\ell}-\dot{k})^{2}+2 \dot{h} L \cos k \cdot \dot{k}\right. \\
& +2 h L \sin k \cdot \dot{k}(\dot{\ell}-\dot{k})+2 \dot{h} L \cos k(\dot{\ell}-\dot{k})] d t \\
& +\left(x_{1}^{2}+x_{2}^{2}\right) \int h^{2}(\dot{\ell}-\dot{k})^{2} d t+\left(x_{1}^{2}+x_{2}^{2}\right) \int \dot{h}^{2} d t+L^{2} \int \dot{\ell}^{2} d t .
\end{aligned}
$$

If we consider Eq. (39) of [8]

$$
U^{\prime}(t)=\left(\begin{array}{l}
u_{1}^{\prime}(t) \\
u_{2}^{\prime}(t)
\end{array}\right)=\left(\begin{array}{l}
L \cos (\ell(t)) \\
L \sin (\ell(t))
\end{array}\right)
$$

and we calculate the time derivative of this equation,

$$
\begin{aligned}
& d u_{1}^{\prime}=-L \sin \ell \cdot \dot{\ell} d t \\
& d u_{2}^{\prime}=L \cos \ell \cdot \dot{\ell} d t
\end{aligned}
$$

is found. Then If Eq. (39) of [8] and Eq. (24) are replaced in calculating data $\dot{x}_{1}^{2}+\dot{x}_{2}^{2}$ and in Section 2 , by using the parameter $d t \rightarrow d a, \dot{a} \rightarrow a^{\prime}=\frac{d a}{d a}=$ 
$1, \int \rightarrow \oint:$

$$
\begin{aligned}
2 S= & \left(x_{1}^{2}+x_{2}^{2}\right) \int_{t_{1}}^{t_{2}} \frac{d h^{2}}{d a}+L^{2} \int_{t_{1}}^{t_{2}} \frac{d \ell^{2}}{d a} \\
& +x_{1} \int_{t_{1}}^{t_{2}}\left[-2 h u_{1} d a-2 \frac{d h}{d a} d u_{1}-2 h d u_{2}+2 u_{2} d h\right] \\
& +x_{2} \int_{t_{1}}^{t_{2}}\left[-2 h u_{2} d a-2 \frac{d h}{d a} d u_{2}+2 h d u_{1}-2 u_{1} d h\right]
\end{aligned}
$$

can be obtained.

If Eq. (18) of [8] is replaced in Eq. (26) and the necessary operations are done,

$$
\begin{aligned}
2 S= & x_{1} \int_{t_{1}}^{t_{2}}-2 h^{2} p_{1} d a+x_{1} \int_{t_{1}}^{t_{2}}\left[-2 h p_{2} d h+h d u_{2}+u_{2} d h\right] \\
& +x_{2} \int_{t_{1}}^{t_{2}}-2 h^{2} p_{2} d a+x_{2} \int_{t_{1}}^{t_{2}}\left[-2 h p_{1} d h-h d u_{1}-u_{1} d h\right] \\
& +\left(x_{1}^{2}+x_{2}^{2}\right) \int_{t_{1}}^{t_{2}} \frac{d h^{2}}{d a}+x_{1} \int_{t_{1}}^{t_{2}}\left[u_{2} d h-h d u_{2}-2 \frac{d h}{d a} d u_{1}\right] \\
& +L^{2} \int_{t_{1}}^{t_{2}} \frac{d \ell^{2}}{d a}+x_{2} \int_{t_{1}}^{t_{2}}\left[-u_{1} d h+h d u_{1}-2 \frac{d h}{d a} d u_{2}\right]
\end{aligned}
$$

is obtained.

If we consider Eqs. (46), (47) of [8]

$$
\begin{gathered}
\oint[\underbrace{-2 h^{2} p_{1} d a-2 h p_{2} d h+h d u_{2}+u_{2} d h}_{a}]=a^{*}, \\
\oint[\underbrace{-2 h^{2} p_{2} d a+2 h p_{1} d h-h d u_{1}-u_{1} d h}_{\mu_{1}}]=b^{*}, \\
2 F=\left(a+\mu_{1}\right) x_{1}+\left(b+\mu_{2}\right) x_{2} .
\end{gathered}
$$


and Eqs. (46) and (47) of [8] are replaced in Eq. (27),

$$
\begin{aligned}
2 S= & 2 F+\left(x_{1}^{2}+x_{2}^{2}\right) \int_{t_{1}}^{t_{2}} \frac{d h^{2}}{d a}+L^{2} \int_{t_{1}}^{t_{2}} \frac{d \ell^{2}}{d a} \\
& +x_{1} \int_{t_{1}}^{t_{2}}\left[u_{2} d h-h d u_{2}-2 \frac{d h}{d a} d u_{1}\right] \\
& +x_{2} \int_{t_{1}}^{t_{2}}\left[-u_{1} d h+h d u_{1}-2 \frac{d h}{d a} d u_{2}\right]
\end{aligned}
$$

is arrived at the relation between the formula of kinetic energy and the area formula for application.

\section{References}

[1] H. Dathe, R. Gezzi, Characteristic directions of closed planar motions, Zeitschrift für Angewandte Mathematik und Mechanik, 92 (2012), 2-13.

[2] H. Dathe, R. Gezzi, Addenda and Erratum to: Characteristic directions of closed planar motions, Zeitschrift für Angewandte Mathematik und Mechanik, 94 (2014), 551-554.

[3] A. Tutar, E. Inan, The formula of kinetic energy for the closed planar homothetic direct motions, International Journal of Applied Mathematics, 28, No. 3 (2015), 213-222.

[4] H.R. Müller, Verallgemeinerung einer Formel von Steiner, Abh. Braunschweig. Wiss. Ges., 29 (1978), 107-113.

[5] H.R. Müller, Über Trägheitsmomente bei Steinerscher Massenbelegung, Abh. Braunschweig. Wiss. Ges., 29 (1978), 115-119.

[6] J. Steiner, Von dem Krümmungs-Schwerpuncte ebener Curven, Journal für die reine und angewandte Mathematik, 21 (1840), 33-63.

[7] A. Tutar, N. Kuruoğlu, The Steiner formula and the Holditch theorem for the homothetic motions on the planar kinematics, Mechanism and Machine Theory, 34 (1998), 1-6. 
[8] A. Tutar, O. Sener, The Steiner formula and the Polar moment of inertia for the closed planar homothetic motions in complex plane, Advances in Mathematical Physics, Accepted. 
